\title{
INCIDÊNCIA, TIPO E NATUREZA DAS LESÕES \\ DOS ATLETAS DO RÚGBI SÃO JOSÉ NA \\ TEMPORADA DE 2014
}

\author{
INCIDENCE, TYPE AND NATURE OF INJURIES ON ATHLETES OF RUGBY SÃO JOSÉ \\ AT THE 2014 SEASON \\ INCIDENCIA, TIPO Y NATURALEZA DE LAS LESIONES DE LOS ATLETAS DE RUGBY \\ SÃO JOSÉ EN LA TEMPORADA 2014
}

Luís Eduardo de Toledo' (Médico)
Benno Ejnisman' (Médico)
Carlos Vicente Andreoli' (Médico)

1. Universidade Federal de São Paulo (UNIFESP-EPM), São Paulo, SP, Brasil.

\section{Correspondência:}

Rua Francisco Ricci, 181, apto 52 A, São José dos Campos, SP, Brasil. 12243-261.

lettoledo@hotmail.com

\section{RESUMO}

Introdução: O rúgbi será incluído novamente como esporte olímpico nos Jogos Olímpicos Rio 2016 e seus atletas apresentam alta incidência de lesões em virtude das características do esporte. Objetivo: Reportar a incidência, local, natureza, tratamento das lesões e o tempo de afastamento de atletas do Rúgbi São José na temporada de 2014 em função das lesões. Métodos: Foi realizado um estudo transversal com 62 atletas do rúgbi, sendo identificados como principais fatores de risco para lesões na temporada de 2014 a existência de lesões prévias, idade, tempo de prática do rúgbi, IMC elevado, posição de jogo, horas de treino semanal e frequência de treinamento em academia. Resultados: As principais lesões encontradas foram lesões articulares nos membros inferiores. Conclusão: Encontramos alta incidência de lesões nos atletas do Rúgbi São José, com predomínio das lesões articulares dos membros inferiores, sendo os principais fatores de risco a idade mais avançada, maior tempo de prática do rúgbi, IMC elevado, maior tempo de treino semanal e hábito de frequentar academia de musculação, além de presença de lesões prévias e a posição de jogo na "linha".

Palavras-chave: rúgbi, traumatismos em atletas, extremidade inferior.

\section{ABSTRACT}

Introduction: Rugby will be included back as an Olympic sport in the Olympics Rio 2016 and athletes show a high incidence of injuries due to the sports' characteristics. Objective: To report the incidence, location, nature, treatment of injuries and the time of withdrawal from sports due to injuries of Rugby São Jose athletes in the 2014 Season. Methods: We conducted a cross-sectional study with 62 rugby athletes identifying the main risk factors for injuries in the 2014 season, the existence of previous injuries, age, time practicing rugby, high BMI, playing position, hours of weekly training and gym training habits. Results: The main injuries found were in lower limbs joints. Conclusion: We found a high incidence of injuries in Rugby São José athletes, with a predominance of joint injuries at the lower limbs, the main risk factors being: age, time playing rugby, high BMI, highest weekly workout frequency and gym training habits, besides the presence of previous injuries and playing in the "in line" position.

Keywords: rugby, athletic injuries, lower extremity.

\section{RESUMEN}

Introducción: El rugby será un deporte olímpico en Rio 2016 y sus atletas poseen una alta incidencia de lesiones debido a las características del deporte. Objetivo: Reportar la incidencia, el local, la naturaleza, el tratamiento y el momento de alejamiento de las lesiones en los atletas de Rugby São José en la temporada 2014. Métodos: Estudio transversal realizado con 62 atletas de rugby, siendo identificados como factores principales de riesgo para lesiones en la temporada 2014, la existencia de lesiones previas, edad, tiempo de rugby, IMC elevado, posición de juego, horas de entrenamiento semanal y frecuencia en gimnasio. Resultados: Las principales lesiones encontradas fueron las articulaciones en los miembros inferiores. Conclusión: Se encontró alta incidencia de lesiones en atletas de Rugby São José, con predominio de lesiones articulares de los miembros inferiores, siendo los principales factores de riesgo la mayor edad, más tiempo de rugby, IMC elevado, mayor tiempo de entrenamiento semanal y hábito de frecuentar gimnasio de musculación, además de la presencia de lesiones anteriores y la posición de juego en la "línea".

Palabras clave: rugby, traumatismos en atletas, extremidad inferior. 


\section{INTRODUÇÃO}

O rúgbi é um esporte que envolve contato físico intenso e exige um condicionamento físico de alto nível. Força e potência muscular, endurance, velocidade, aceleração e agilidade são requisitos importante para os atletas. Por essas características, é um esporte que tem uma alta incidência de lesões, principalmente nas ligas amadoras e semi-profissionais dos Estados Unidos e Europa ${ }^{1,2}$. Mas nos países onde a sua prática é bastante difundida, com a profissionalização das ligas e programas de prevenção de lesão, como na Nova Zelândia (The New Zeland Rugby Smart Programme) e Austrália, a incidência das lesões é bastante estudada' e os fatores de risco associados já estabelecidos ${ }^{1,2}$.

A maioria das lesões no rúgbi é de natureza músculo-tendínea e afetam os membros inferiores ${ }^{3,4}$, sendo o tackle a fase do jogo mais relacionada às lesões ${ }^{2}$, mas o jogo aberto também tem sua incidência relatada. Índice de Massa Corporal (IMC) elevado, tempo de prática do rúgbi, posição do jogador, relação entre horas de treino e jogo por semana, assim como lesões prévias ${ }^{2,5,6}$, são fatores citados na literatura mundial.

O rúgbi será esporte olímpico pela primeira vez no Rio 2016 e registra um aumento significativo do número de praticante e do número de times no Brasil nos últimos anos. Por se tratar de um esporte relativamente novo no país, carece de estudos relacionados à incidência e natureza das lesões nos atletas brasileiros ${ }^{1}$, assim como programas de prevenção de lesões na modalidade.

O propósito deste estudo foi reportar a incidência, o local, a natureza, o tratamento e o tempo de afastamento das lesões nos atletas do Rúgbi São José na temporada de 2014.

\section{MATERIAL E MÉTODOS}

Foi realizado um estudo transversal com aplicação de um questionário antes do treino para 62 atletas do São José Rúgbi, São José dos Campos, Brasil, time por oito vezes Campeão Nacional, para as categorias Adulto, M17 (menor de 17 anos) e M19 (menor de 19 anos), sendo 51 atletas masculinos e 11 femininos. As principais variáveis avaliadas no questionário foram idade, categoria, gênero, posição no jogo, IMC (Índice de Massa Corporal), tempo de rúgbi, lesão na temporada (2014) e na passada, tempo de afastamento em 2014, tipo, localização e tratamento das lesões. Além do hábito dos atletas de frequentarem academia de musculação e de realizarem um trabalho de prevenção de lesão. A análise estatística de todas as informações coletadas foi inicialmente feita de forma descritiva através da média, mediana, valores mínimo e máximo, desvio-padrão, frequências absoluta e relativa (porcentagem), gráficos de dispersão unidimensional, barras e setor circular?.

As análises inferenciais empregadas com o intuito de confirmar ou refutar evidências encontradas na análise descritiva foram:

Qui-quadrado de Pearson ou Exato de Fisher ${ }^{8}$ na comparação dos grupos com e sem lesão, segundo gênero, classe de índice de massa corporal, categoria etária e posição no jogo, presença de lesão em temporadas anteriores e hábito de prevenir lesão e frequentar academia

Mann-Whitney ${ }^{9}$ na comparação dos grupos com e sem lesão, segundo idade (anos), tempo de rúgbi (meses) e horas de treino por semana

Em todas as conclusões obtidas através das análises inferenciais foi utilizado o nível de significância a igual a 5\%.

Os dados foram digitados em planilhas do Excel 2010 for Windows para o adequado armazenamento das informações. As análises estatísticas foram realizadas com o programa estatístico $R$ versão 2.15.2.

\section{RESULTADOS}

A amostra selecionada nesta pesquisa foi composta por 62 atletas de rúgbi do time do São José, sendo que 35 (56,5\%) sofreram alguma lesão na temporada de 2014 e o restante, 27 (43,5\%) atletas não sofreram lesão nessa temporada (tabela 1).

O grupo dos 35 atletas que sofreram lesão foi composto por 28 $(80,0 \%)$ homens e sete $(20,0 \%)$ mulheres. A idade média deste grupo foi de 20,5 anos, variando de 14 a 39 anos, com desvio-padrão de 6,3 anos (figura 1). Considerando a classe do índice de massa corporal (IMC), temos que 15 (42,9\%) foram classificados como normais, 19 (54,3\%) estavam acima do peso e apenas um (2,9\%) atleta foi considerado obeso (figura 2).

Nesse grupo com lesão, 13 (37,1\%) atletas pertenciam a categoria etária até 17 anos (M17), 7 (20,0\%) eram da categoria até 19 anos (M19) e 15 (42,9\%) eram da categoria adulto (figura 3). Com respeito a posição destes atletas no jogo, 22 (62,9\%) jogavam na posição"linha"e 13 (37,1\%) jogavam na posição "scrum" (figura 4). O tempo mediano de tempo de rúgbi destes atletas foi de 60 meses, variando de 12 a 276 meses (figura 5).

Tabela 1. Características gerais dos atletas, segundo presença de lesão na temporada de 2014

\begin{tabular}{|c|c|c|c|c|c|c|c|}
\hline & \multicolumn{4}{|c|}{ Lesão na temporada de 2014} & \multirow{2}{*}{\multicolumn{2}{|c|}{$\begin{array}{c}\text { Total } \\
(n=62)\end{array}$}} & \\
\hline & \multicolumn{2}{|c|}{$\operatorname{Sim}(n=35)$} & \multicolumn{2}{|c|}{ Não (n=27) } & & & $\mathbf{p}$ \\
\hline \multicolumn{8}{|l|}{ Gênero } \\
\hline Masculino & 28 & $80,0 \%$ & 22 & $81,5 \%$ & 50 & $80,6 \%$ & $0,884^{\mathrm{a}}$ \\
\hline Feminino & 7 & $20,0 \%$ & 5 & $18,5 \%$ & 12 & $19,4 \%$ & \\
\hline \multicolumn{8}{|l|}{ Idade (Anos) } \\
\hline Média & \multicolumn{2}{|r|}{20,5} & \multicolumn{2}{|r|}{18,1} & \multicolumn{2}{|r|}{19,5} & $0,017^{b}$ \\
\hline Mediana & \multicolumn{2}{|r|}{17,0} & \multicolumn{2}{|r|}{16,0} & \multicolumn{2}{|r|}{17,0} & \\
\hline Mínimo-máximo & \multicolumn{2}{|c|}{$14-39$} & \multicolumn{2}{|r|}{$14-40$} & \multicolumn{2}{|r|}{$14-40$} & \\
\hline Desvio-padrão & \multicolumn{2}{|r|}{6,3} & \multicolumn{2}{|r|}{5,8} & \multicolumn{2}{|r|}{6,2} & \\
\hline \multicolumn{8}{|l|}{ Classe de IMC } \\
\hline Normal & 15 & $42,9 \%$ & 15 & $55,6 \%$ & 30 & $48,4 \%$ & $0,021^{c}$ \\
\hline Acima do peso & 19 & $54,3 \%$ & 7 & $25,9 \%$ & 26 & $41,9 \%$ & \\
\hline Obeso & 1 & $2,9 \%$ & 5 & $18,5 \%$ & 6 & $9,7 \%$ & \\
\hline \multicolumn{8}{|l|}{ Categoria } \\
\hline Até 17 anos & 13 & $37,1 \%$ & 14 & $51,9 \%$ & 27 & $43,5 \%$ & $0,126^{a}$ \\
\hline Até 19 anos & 7 & $20,0 \%$ & 8 & $29,6 \%$ & 15 & $24,2 \%$ & \\
\hline Adulto & 15 & $42,9 \%$ & 5 & $18,5 \%$ & 20 & $32,3 \%$ & \\
\hline \multicolumn{8}{|l|}{ Posição } \\
\hline Linha & 22 & $62,9 \%$ & 12 & $44,4 \%$ & 34 & $54,8 \%$ & $0,149^{a}$ \\
\hline Scrum & 13 & $37,1 \%$ & 15 & $55,6 \%$ & 28 & $45,2 \%$ & \\
\hline \multicolumn{8}{|c|}{ Tempo de rúgbi (meses) } \\
\hline Média & \multicolumn{2}{|r|}{82,1} & \multicolumn{2}{|r|}{53,2} & \multicolumn{2}{|r|}{69,5} & $0,027^{b}$ \\
\hline Mediana & & 60,0 & & 36,0 & & 48,0 & \\
\hline Mínimo-máximo & & $2-276$ & & $3-288$ & & $3-288$ & \\
\hline Desvio-padrão & & 71,4 & & 58,6 & & 67,2 & \\
\hline Lesões Prévias & & & & & & & \\
\hline Sim & 19 & $54,3 \%$ & 7 & $25,9 \%$ & 26 & $41,9 \%$ & $0,025^{a}$ \\
\hline Não & 16 & $45,7 \%$ & 20 & $74,1 \%$ & 36 & $58,1 \%$ & \\
\hline Previne Lesão & & & & & & & \\
\hline $\operatorname{Sim}$ & 10 & $28,6 \%$ & 2 & $7,4 \%$ & 12 & $19,4 \%$ & $0,036^{a}$ \\
\hline Não & 25 & $71,4 \%$ & 25 & $92,6 \%$ & 50 & $80,6 \%$ & \\
\hline Frequenta Academi & & & & & & & \\
\hline Sim & 31 & $88,6 \%$ & 19 & $70,4 \%$ & 50 & $80,6 \%$ & $0,072^{a}$ \\
\hline Não & 4 & $11,4 \%$ & 8 & $29,6 \%$ & 12 & $19,4 \%$ & \\
\hline Horas de treino por & & & & & & & \\
\hline Média & & 8,1 & & 6,0 & & 7,2 & $0,003^{b}$ \\
\hline Mediana & & 8,0 & & 4,0 & & 6,0 & \\
\hline Mínimo-máximo & & 4-14 & & 4-14 & & 4-14 & \\
\hline Desvio-padrão & & 2,8 & & 3,2 & & 3,1 & \\
\hline
\end{tabular}




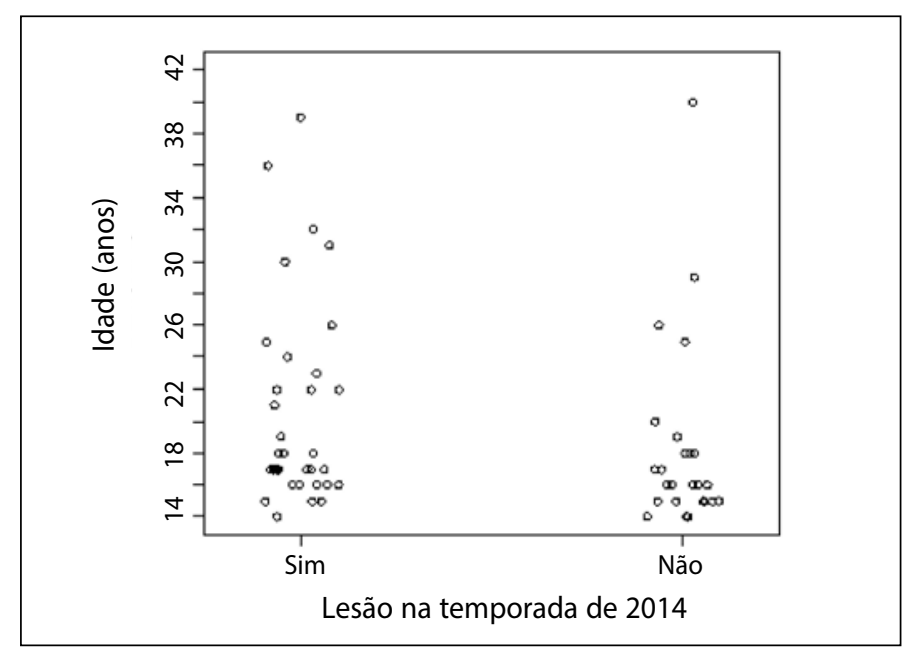

Figura 1. Diagrama de dispersão unidimensional da idade (anos) dos atletas, segundo presença de lesão na temporada de 2014.

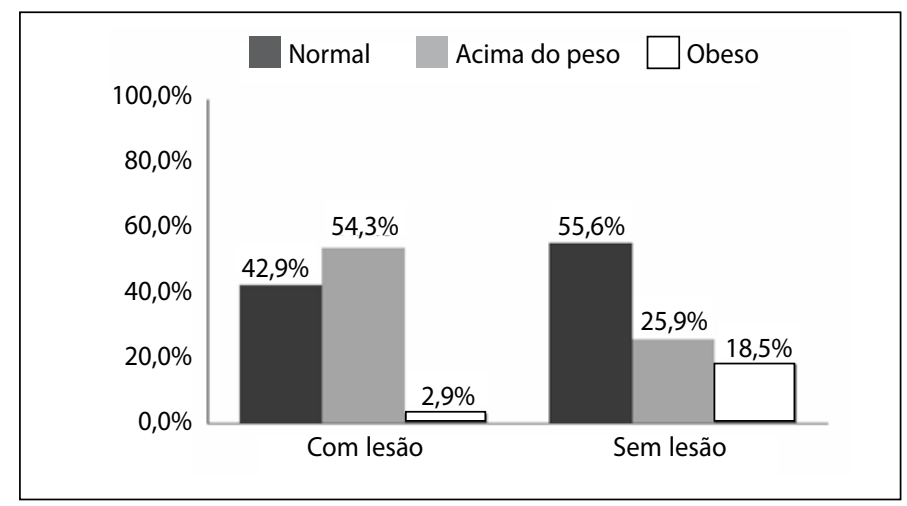

Figura 2. Distribuição da classe do índice de massa corporal dos atletas, segundo presença de lesão na temporada de 2014

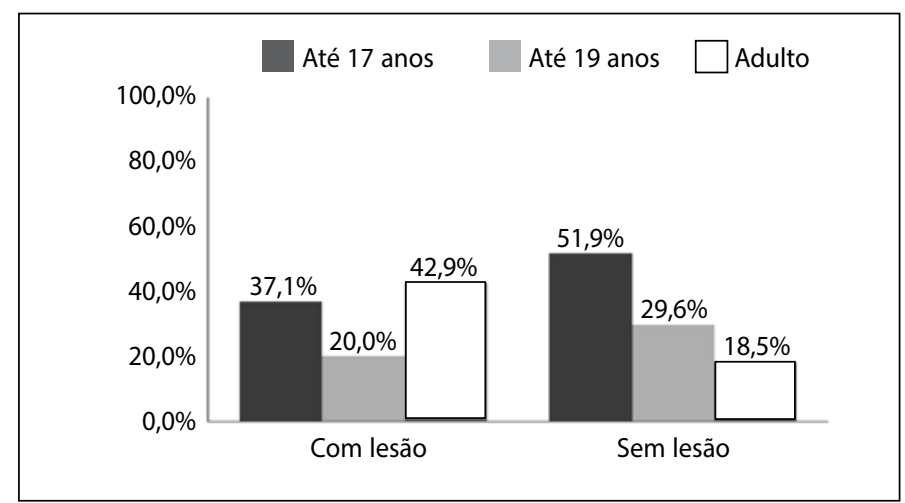

Figura 3. Distribuição da categoria etária dos atletas, segundo presença de lesão na temporada de 2014.

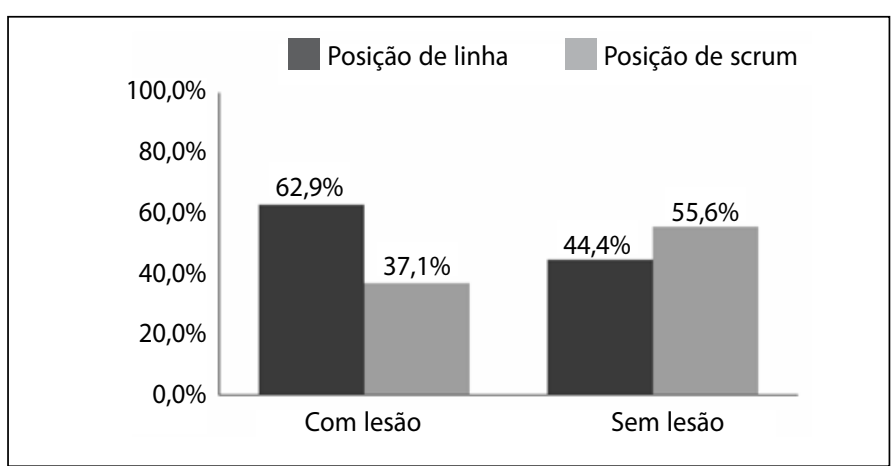

Figura 4. Distribuição da posição durante o jogo dos atletas, segundo presença de lesão na temporada de 2014

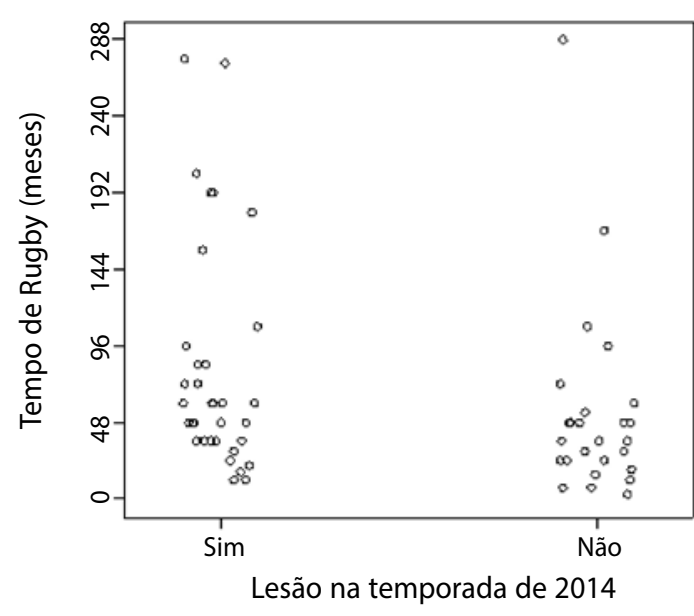

Figura 5. Diagrama de dispersão unidimensional do tempo de rugby (meses) dos atletas, segundo presença de lesão na temporada de 2014.

Cerca de 19 (54,3\%) atletas já haviam tido alguma lesão em temporadas anteriores a 2014 e 10 (28,6\%) relataram o hábito de preveni-las. A maioria dos atletas desse grupo, cerca de 31 (88,6\%) atletas, frequentam academia de maneira regular. Em média estes atletas treinam por semana cerca de 8,1 horas, variando de 4 a 14 horas, com desvio-padrão de 2,8 horas.

O grupo dos 27 atletas que não sofreram lesão na temporada de 2014 foi composto por 22 (81,5\%) homens e cinco (18,5\%) mulheres. A idade média deste grupo foi de 18,1 anos, variando de 14 a 40 anos, com desvio-padrão de 5,8 anos. Considerando a classe do IMC, temos que 15 (55,6\%) foram classificados como normais, sete $(25,9 \%)$ estavam acima do peso e cinco (18,5\%) atletas foram considerados obesos.

No grupo sem lesão, 14 (51,9\%) atletas pertenciam a categoria M17, oito $(29,6 \%)$ eram da categoria M19 e cinco (18,5\%) eram da categoria adulto. Com respeito a posição destes atletas no jogo, 12 (44,4\%) jogavam na posição "linha" e 15 (55,6\%) jogavam na posição "scrum". O tempo mediano de tempo de rúgbi destes atletas foi de 36 meses variando de 3 a 288 meses. Cerca de sete $(25,9 \%)$ atletas já haviam tido alguma lesão em temporadas anteriores a 2014 e dois $(7,4 \%)$ relataram o hábito de preveni-las. A maioria dos atletas desse grupo, cerca de 19 $(70,4 \%)$ atletas, frequentam academia de maneira regular. Em média estes atletas treinam por semana cerca de 6,0 horas, variando de 4 a 14 horas, com desvio-padrão de 3,2 horas.

Os resultados inferenciais revelaram que os grupos que sofreram e não sofreram leão tem o mesmo perfil quanto ao gênero $(p=0,884)$, categoria etária $(p=0,126)$ e posição no jogo $(p=0,149)$.

O grupo que sofreu lesão em 2014 apresentou maior idade $(p=0,017)$, tempo de rúgbi $(p=0,027)$ e horas de treino por semana $(p=0,003)$ quando comparado ao grupo que não sofreu lesão. Este grupo com lesão também apresentou mais atletas acima do peso ( $p=0,021)$, lesões prévias $(p=0,025)$ e hábito de prevenir lesão $(p=0,036)$ com mais frequência quando comparado ao grupo sem lesão.

É importante ressaltar a tendência dos atletas do grupo com lesão em utilizar academia com mais frequência quando comparado ao grupo sem lesão $(p=0,072)$.

Foi também importante objeto de investigação desta pesquisa a descrição detalhada do tipo, localização e número de lesões, tratamento seguido e tempo de afastamento dos atletas que sofreram lesão (tabela 2).

Os tipos de lesões mais frequentes entre os 35 atletas que sofreram lesão na temporada de 2014 foram a lesão articular em 11 (31,4\%) atletas, lesão muscular em 10 (28,6\%) atletas, fratura em nove (25,7\%) atletas e contusão em cinco (14,3\%) deles. 
Tabela 2. Distribuição do tipo, localização e número de lesões, tratamento seguido e tempo de afastamento dos atletas que sofreram lesão na temporada de 2014.

\begin{tabular}{|c|c|c|c|}
\hline \multirow[t]{5}{*}{ Tipo de lesão } & Articular & 11 & $31,4 \%$ \\
\hline & Muscular & 10 & $28,6 \%$ \\
\hline & Fratura & 9 & $25,7 \%$ \\
\hline & Contusão & 5 & $14,3 \%$ \\
\hline & Total & 35 & $100,0 \%$ \\
\hline \multirow[t]{5}{*}{ Localização da lesão } & Membro inferior & 16 & $45,7 \%$ \\
\hline & Membro superior & 12 & $34,3 \%$ \\
\hline & Ambos os membros & 4 & $11,4 \%$ \\
\hline & Outra região & 3 & $8,6 \%$ \\
\hline & Total & 35 & $100,0 \%$ \\
\hline \multirow[t]{4}{*}{ Número de lesões } & Uma & 23 & $65,7 \%$ \\
\hline & Duas & 8 & $22,9 \%$ \\
\hline & Três & 4 & $11,4 \%$ \\
\hline & Total & 35 & $100,0 \%$ \\
\hline \multirow[t]{9}{*}{ Tratamento } & Fisioterapia & 13 & $37,1 \%$ \\
\hline & Cirurgia+fisioterapia & 6 & $17,1 \%$ \\
\hline & Imobilização+fisioterapia & 4 & $11,4 \%$ \\
\hline & Fisioterapia+medicação & 3 & $8,6 \%$ \\
\hline & Imobilização & 2 & $5,7 \%$ \\
\hline & Imobilização+fisioterapia & 1 & $2,9 \%$ \\
\hline & Medicação & 1 & $2,9 \%$ \\
\hline & Desconhecido & 5 & $14,3 \%$ \\
\hline & Total & 35 & $100,0 \%$ \\
\hline \multirow[t]{4}{*}{ Tempo de afastamento (semanas) } & Média & & 9,6 \\
\hline & Mediana & & 5,0 \\
\hline & Mínimo-máximo & & $1-96$ \\
\hline & Desvio-padrão & & 16,2 \\
\hline
\end{tabular}

Quanto a localização, temos que $16(45,7 \%)$ atletas tiveram lesão apenas no membro inferior, 12 (34,3\%) tiveram lesão apenas no membro superior, quatro $(11,4 \%)$ tiveram lesão em ambos os membros e três $(8,6 \%)$ atletas tiveram lesão em outra região do corpo.

O número de lesões sofridas por estes atletas variou de um a três, sendo que $23(65,7 \%)$ atletas sofreram apenas uma lesão, oito $(22,9 \%)$ sofreram duas lesões e quatro $(11,4 \%)$ atletas sofreram três lesões.

O tratamento mais recomendado e seguido pelos atletas foi a fisioterapia, por $13(37,1 \%)$ atletas. A cirurgia acompanhada da fisioterapia foi recomendada para seis $(17,1 \%)$ atletas. Cerca de quatro $(11,4 \%)$ atletas fizeram imobilização mais fisioterapia. A fisioterapia agregada a medicação foi recomendada a três (8,6\%) atletas. A imobilização foi tratamento seguido por dois (5,7\%) atletas, enquanto que imobilização acompanhada da fisioterapia foi tratamento sugerido para um $(2,9 \%)$ atleta. O mesmo ocorreu com a medicação, adotado também por apenas um (2,9\%) atleta. Vale ressaltar que, para cinco (14,3\%) atletas, não foi possível o registro do tipo de tratamento utilizado na lesão. A maioria dos atletas ficou até 24 semanas afastados dos jogos e apenas um atleta apresentou tempo maior de afastamento, ficando 96 semanas afastado.

\section{DISCUSSÃO}

No grupo dos atletas que sofreram lesão na temporada de 2014 (56,5\%) encontramos resultados aumentados nos principais fatores de risco citados na literatura mundial $4,10,11$, quando comparados aos atletas sem lesão em 2014 (43,5\%).

A maior idade encontrada no grupo com lesão (figuras 1), assim como o maior tempo de rúgbi (figura 5) e o maior número de horas semanais de treino (figura 6 ) nos atletas lesionados em 2014 , respectivamente $(p=0,017),(p=0,027)$ e $(p=0,003)$, encontram correlação com os achados dos trabalhos realizados nas ligas da Nova Zelândia e Austrália 2,3.

Quanto mais idoso, quanto mais horas treino semanal ${ }^{12}$ e mais tempo de rúgbi o atleta tiver, mais lesões aparecem, mostrando que a experiência do atleta não é capaz de diminuir suas chances de lesão.

Além disso, ao analisarmos a existência de lesões prévias como fator de risco para lesão em 2014, também constatamos uma maior incidência no grupo dos atletas que sofreram lesão em 2014 (figura 7).

Cerca de 54,3\% dos atletas que sofreram lesão em 2014 já tiveram lesão na temporada anterior, contra 25,9\% dos atletas que não tiveram lesão em 2014, mas tiveram lesões na temporada passada ( $p=0,025)$. Ou seja, uma vez lesionado, aumenta-se a possibilidade de novas lesões.

O IMC dos atletas também se mostrou um bom preditor de lesão no presente estudo. Encontramos uma maior prevalência de atletas acima do peso $(54,3 \%)$ no grupo com lesão, do que no grupo sem lesão, 25,9\% respectivamente ( $p=0,021)$ (figura 2$)$.

Outro dado significativo é a maior tendência dos atletas que sofreram lesão na temporada de 2014 de frequentar academia e musculação $(p=0,072)$ (figura 8).

Exercícios para hipertrofia muscular são tidos como benéficos para melhorar a performance e a força dos atletas do rúgbi. Porém, no caso específico do presente estudo, é preciso ficar atento ao volume total de treino semanal destes atletas, somado ao tempo dispendido na academia. Fadiga e overtraining devem ser investigados e um melhor equilíbrio no volume de treino semanal deve ser objetivado.

Os atletas que sofreram lesão em 2014 se preocupam mais com o hábito de prevenir lesão do que o grupo que não sofreu lesão $(p=0,036)$.

Possíveis explicações para este contrassenso, podem estar relacionadas ao fato destes mesmos atletas terem uma maior prevalência de lesões prévias, portanto, já realizaram tratamentos que motivaram o trabalho preventivo.

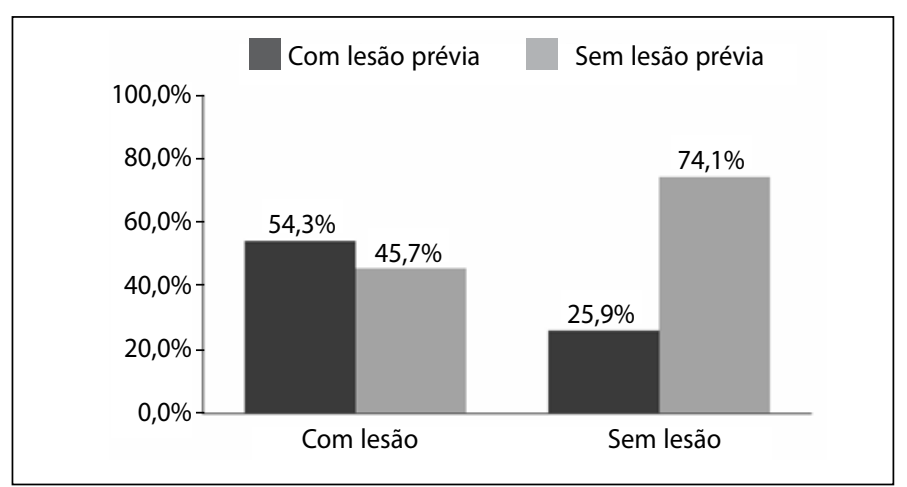

Figura 6. Distribuição da presença de lesão prévia entre os atletas, segundo presença de lesão na temporada de 2014.

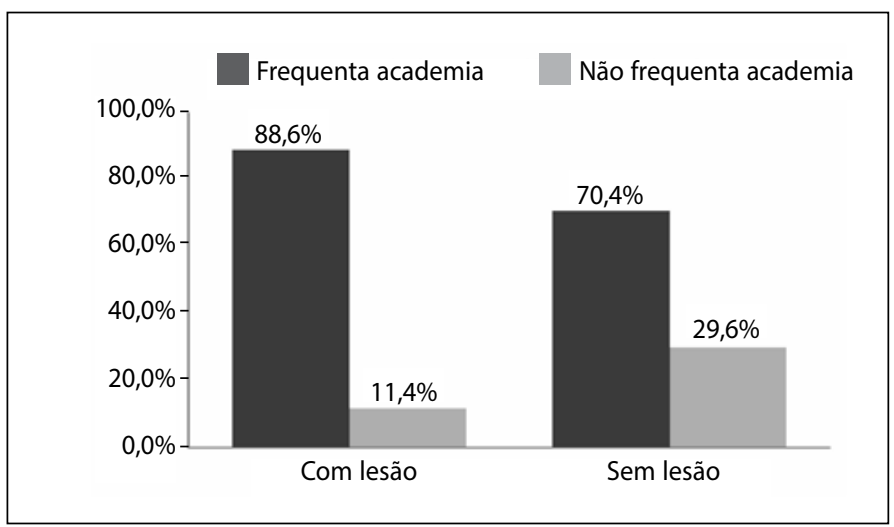

Figura 7. Distribuição do hábito de frequentar academia entre os atletas, segundo presença de lesão na temporada de 2014. 
Outro fator importante é avaliar através de futuros estudos a qualidade desse trabalho preventivo e sua eficácia na prevenção de lesões para o rúgbi, nos moldes do que é feito no futebol com o FIFA $11+$.

Os atletas que mais sofreram lesão em 2014 foram os da "linha" (62,9\%) em relação à posição "scrum" (37,1\%).

A lesão mais frequente foi a que acometeu o membro inferior, com $45,7 \%$ das lesões, contra 34,3\% do membro superior. Lesões em ambos os membros 11,4\% e outras regiões 8,6\% (figura 9). Esses dados condizem com a literatura que aponta os membros inferiores como os mais lesionados no Rugby $1-4,12-14$.

As principais lesões são as articulares (31,4\%), musculares (28,6\%), fraturas $(25,7 \%)$ e contusões (14,3\%) (figura 10).

Baseado nesses achados, podemos orientar atletas e comissão técnica a formularem protocolos de aquecimento e treinamento proprioceptivo, que visem a prevenção das lesões mais prevalentes no Rúgbi São José.

Dessa forma, pretende-se diminuir o número de lesões dos atletas, o tempo de afastamento dos mesmos, que ficam em média de 5 à 20 semanas afastados por lesão e a gravidade das lesões, quando analisamos os tratamentos instituídos (tabela 2).

Em relação ao tratamento, vemos que as lesões tiveram um moderado grau de gravidade, já que 37,1\% dos atletas foram submetidos à fisioterapia e 17,1\% à cirurgia e fisioterapia, além de outras combinações de tratamento (tabela 2).

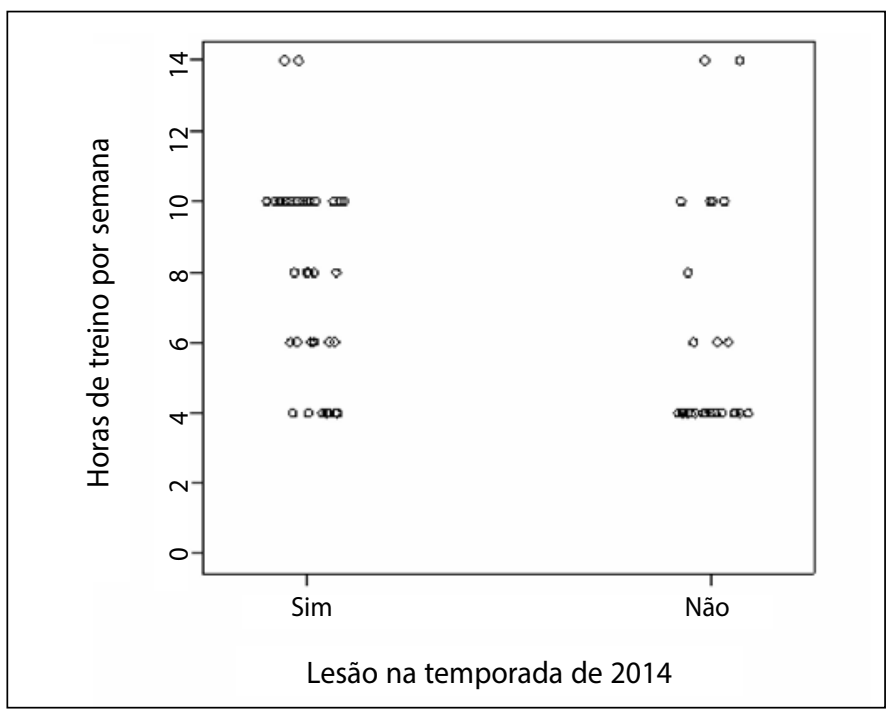

Figura 8. Diagrama de dispersão unidimensional das horas de treino por semana dos atletas, segundo presença de lesão na temporada de 2014.

\section{REFERÊNCIAS}

1. Gabbett TJ. Incidence, site, and nature of injuries in amateur rugby league over three consecutive seasons. Br J Sports Med. 2000;34(2):98-103.

2. Quarrie KL, Alsop JC, Waller AE, Bird YN, Marshall SW, Chalmers DJ. The New Zealand rugby injury and performance project. VI. A prospective cohort study of risk factors for injury in rugby union football. Br J Sports Med. 2001;35(3):157-66

3. Hoskins W, Pollard H, Hough K, Tully C. Injury in rugby league. J Sci Med Sport. 2006;9(1-2):46-56

4. Kaplan KM, Goodwillie A, Strauss EJ, Rosen JE. Rugby injuries: a review of concepts and current literature. Bull NYU Hosp Jt Dis. 2008;66(2):86-93.

5. Brooks JH, Kemp SP. Injury-prevention priorities according to playing position in professional rugby union players. Br J Sports Med. 2011;45(10):765-75.

6. Junge A, Cheung K, Edwards T, Dvorak J. Injuries in youth amateur soccer and rugby players--comparison of incidence and characteristics. Br J Sports Med. 2004;38(2):168-72.

7. Morettin PA, Bussab WO. Estatística básica. 5a. ed. São Paulo: Saraiva; 2002.

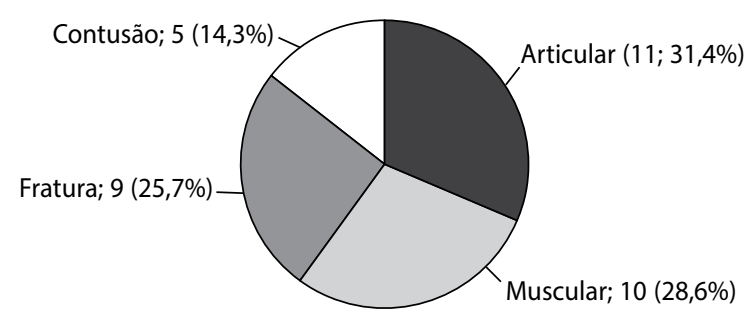

Figura 9. Distribuição do tipo de lesão sofrida pelos atletas lesionados na temporada de 2014 .

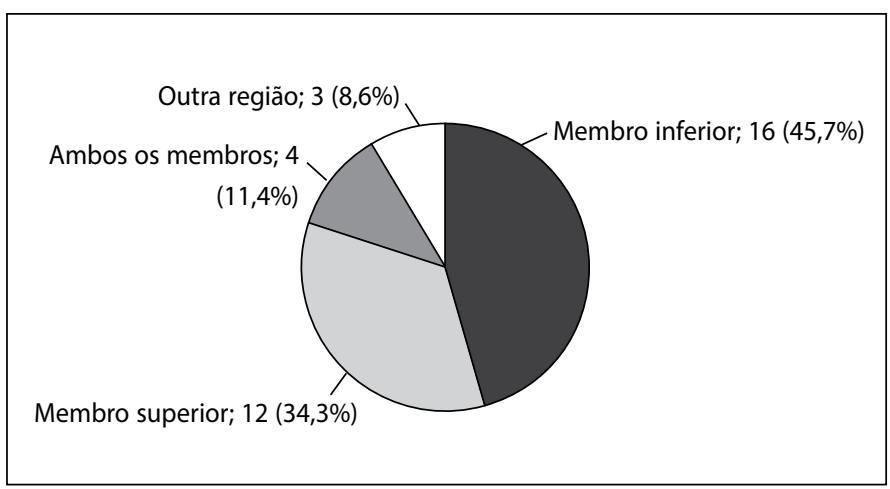

Figura 10. Distribuição da localização da lesão sofrida pelos atletas lesionados na temporada de 2014.

\section{CONCLUSÃO}

Foram encontrados dados que demonstram uma alta incidência de lesões nos atletas do Rúgbi São José na temporada de 2014, sendo identificados como principais fatores de risco para lesão a maior idade, maior tempo de rúgbi, IMC elevado, maior tempo de treino semanal e hábito de frequentar academia de musculação, além de lesões prévias e a posição de jogo na "linha".

Os membros inferiores foram os mais lesionados, sendo as lesões articulares as mais frequentes.

Recomendamos que ações visando a prevenção das lesões devam ser estudadas e intensificadas nos atletas praticantes do rúgbi.

Todos os autores declararam não haver qualquer potencial conflito de interesses referente a este artigo. 